
Military Technical College, Kobry El-Kobbah, Cairo, Egypt

$9^{\text {th }}$ International Conference

On Aerospace Sciences \& Aviation Technology

\title{
ANALYSIS OF JAMMING TECHNIQUE AGAINST IAC MONOPULSE RADAR
}

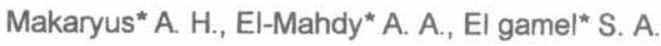

\section{ABSTRACT}

This paper presents the mathematical analysis of a proposed jamming technique used against IAC monopulse radar. The idea of this technique is to repeat the radar signal with change in its amplitude and phase (deception jamming signal) to produce an angular error in the target tracker. This error changes for different values of amplitude ratio and phase difference w.r.t the target signal. In the generated deceptive jamming signals, the change in the amplitude ratio makes large effect than the change in the phase difference on the real part of the complex measured error angle. The real value of the complex measured angle due to jamming with constant phase difference and different amplitude ratio has large variation at amplitude ratio less than three. This value converges to the value of the angle of the false target for higher amplitude ratio. There is no jamming effect at amplitude ratios less than one and for some values of phase difference. At these values, the measured angle equals to the angle of the real target. At least two jamming signals are required to have the complex error jamming angle to overcome the ECCM techniques that might be used by the target.

KEY WORDS: Jamming, and IAC monopulse radar

- Egyptian Armed Forces 


\section{Introduction}

Monopulse radars are commonly used in target tracking because of their angular accuracy. These radars provide a better angular accuracy and less sensitivity to fluctuation in the radar cross section (RCS) of the target compared with other types of tracking radars (such as sequential lobbing and conical scan). In practice these radars use four lobes (two four elevation angle measurements and the others for azimuth angle). The echo received by the four lobes are mixed in a hybrid junction to provide three signals, namely the summation and two difference (in azimuth and in elevation). The target angle with respect to (w.r.t) the radar axis is measured via computation of variable named monopulse ratio, which is the ratio of the two difference signals $\left(D_{x}, D_{y}\right)$ and the sum signal $S$ (in the phasor form) $[1,2]$.

This paper is organized as follows. Section II represents the mathematical formulation of the proposed jammer. The Mathematical Analysis of the Proposed Jamming Technique is discussed in section III. Section IV represents the Performance of the proposed Jamming technique Under Possible ECCM Techniques. In Section $\mathrm{V}$ the conclusion of this paper is introduced.

\section{Mathematical Formulation of the Proposed Jammer}

The proposed jamming signal consists of composite CW pulsed signals with different amplitudes, phases, and delays; the generated jamming signal has the following form

$$
S_{j}=\sum_{n=1}^{N} A_{n} \cos \left[\omega_{c}\left(t+\tau_{n}\right)+\varphi_{n}\right], \quad 0 \prec t \prec \delta
$$

where $S_{j}$ is the jammer transmitted signal, $A_{n}$ is the amplitude of the nth $\mathrm{CW}$ jamming signal, $\omega_{c}$ is the angular carrier frequency, $\tau_{n}$ is the relative time delay (relative to the missile signal), and $\varphi_{n}$ is the relative phase difference. The effect of this composite jamming signal appears as multiple false targets, each false target corresponds to one of the $\mathrm{CW}$ jamming signals. These false targets lie in the same range gate of the missile tracking radar. The total effect of this action is that these targets appear as one target different from the real target. The idea of the proposed jamming technique is to introduce an angular error in the target measured angle in IAC monopulse tracking radar. The mathematical formulation of the measured target angle under jamming is expressed as

$$
\frac{1}{\rho} \frac{D}{S}=\theta_{j}=\frac{\theta_{o}+\theta_{1} g_{1} e^{j \phi_{1}}+\ldots \ldots \ldots \ldots \ldots+\theta_{N} g_{N} e^{j \phi_{N}}}{1+g_{1} e^{j \phi_{1}}+\ldots \ldots \ldots \ldots \ldots . .+g_{N} e^{j \phi_{N}}}
$$


where $\theta_{j}$ is the new complex measured angle due to jamming. This angle results from real target (at angle $\theta_{o}$ ) and $N$ false unresolved targets at angles $\theta_{1}, \theta_{2}, \ldots \ldots, \theta_{N}$.

\section{Mathematical Analysis of the Proposed Jamming Technique}

We start the analysis of the mathematical results given in (2) by considering $n=1$, then we can write the complex angle $\left(\theta_{j}\right)$ in the following form

$$
\theta_{j}=\frac{\theta_{0}+\theta_{1} g_{1} e^{j \phi_{1}}}{1+g_{1} e^{j \phi_{1}}}
$$

recall that $\theta_{o}$ is the angle of the real target and $\theta_{1}$ is the angle of a false target. The angles $\theta_{o}$ and $\theta_{1}$ must lie inside the half power beam width (HPBW) of the missile pattern. The considered value of HPBW is equal $1^{\circ}$. For analysis purposes, we assume some values for the angles $\theta_{o}$ and $\theta_{1}$ that lie in HPBW. These values are $0.1^{\circ}$ and $0.5^{\circ}$ for $\theta_{o}$ and $\theta_{1}$ respectively. As shown in Fig.1, the graphical representation of the measured complex angle $\left(\theta_{j}\right)$ for fixed value of $g_{1}$ and varying the phase difference $\phi_{1}$ from $\left(0^{\circ} \rightarrow 360^{\circ}\right)$ represents a circle in the complex plane. Varying the values of $g_{1}$ result in multiple circles. The centers of these circles always lie on the real axis. The circles for $g_{1} \prec 1$ lie in the left hand side of the imaginary axis (the midpoint of $\theta_{o}$ and $\theta_{1}$ ). As $g_{1}$ decreases, we have smaller circles that approach to $\theta_{o}$. When $g_{1}$ reaches zero the resulting circle becomes a point in the real axis, which is $\theta_{o}$. The circles for $g_{1} \succ 1$ lie in the right hand side of the imaginary axis. As $g_{1}$ increases, we have smaller circles that approach to $\theta_{1}$. When $g_{1}$ is a very large value ( $\approx$ infinity), the resulting circle becomes a point in the real axis which is $\theta_{1}$. At $g_{1}=1$, the complex angle $\theta_{j}$ has no real value, it is represented by an imaginary line (because the imaginary axis is shifted to the midpoint of $\theta_{o}$ and $\theta_{1}$ ) as shown in Fig.1.

Moreover, if the values of $g_{1}$ is varying from $(0 \rightarrow \infty)$, for fixed value of the phase difference $\phi_{1}$, we have a group of circles that move along the imaginary axis and always pass through the two angles $\theta_{o}$ and $\theta_{1}$ in the real axis as shown in Fig.2.

In Fig.3, the measured value of $\theta_{j}$ is the intersection of two circles. The first circle (bold one) has a constant $g_{1}$ (equal to 1.5 ) and varying $\phi_{1}$ from $\left(0^{\circ} \rightarrow 360^{\circ}\right)$, and 
the second circle (thin one) has constant $\phi_{1}$ (equal to $30^{\circ}$ ) and varying $g_{1}$ from $(0 \rightarrow \infty)$. Similar results can be obtained by increasing $n$ in (2) as shown in Fig. 4 . This figure shows that for $n=2$, the value of $\theta_{j}$ is the intersection of four circles (two bold circles for constant $g_{1}$ and $g_{2}$ and varying $\phi_{1}$ and $\phi_{2}$ from $\left(0^{\circ} \rightarrow 360^{\circ}\right)$, and two thin circles for constant $\phi_{1}$ and $\phi_{2}$ and varying $g_{1}$ and $g_{2}$ from $(0 \rightarrow \infty)$.

Generally for $n=N$, we have $2 N$ circles and the value of $\theta_{j}$ is the intersection of all of them.

Fixed amplitude ratio $g_{1}$ and different values of phase difference $\phi_{1}$ result in different values of the angle $\theta_{j}$ (intersection in points a1, b1, c1, d1) as shown in Fig.5. Fixed phase difference $\phi_{1}$ and different amplitude ratio $g_{1}$ results in different values for the angle $\theta_{j}$ (intersection in points a2, b2, c2, d2) as shown in Fig.6. From Figs 5 and 6 , we can conclude that the variation of $\phi_{1}$ at fixed $g_{1}$ provides smaller effect on the angle $\theta_{j}$ than varying $g_{1}$ at fixed $\phi_{1}$.

Another way to study (2) is to plot the amplitude ratio $g_{1}$ versus the measured angle $\theta_{j}$ for different phase difference. This plot is shown in Fig.7, a zoom in on this figure is shown in Fig.7a. This figure shows that the same $\theta_{j}$ is obtained for $\phi_{1}$ and $\left(360^{\circ}-\phi_{1}\right)$, so it's enough to change $\phi_{1}$ from $\left(0^{\circ} \rightarrow 180^{\circ}\right)$ to have a change in $\theta_{j}$. All curves converge to $\theta_{j}=0.5$ for high $g_{1}$ (they tend to reach 0.5 for $g_{1} \succ 10$ ) and for small value of $g_{1}\left(g_{1} \prec 3\right)$; we have high change in the angle $\theta_{j}$.

The relation between the phase differences $\phi_{1}$ versus the measured angle $\theta_{j}$ for different values of $g_{1}$ is shown in Fig.8. This figure shows that, for all phase difference from $\left(0^{\circ} \rightarrow 360^{\circ}\right)$ at $g_{1}=0$, the angle $\theta_{j}=0.1$ (equal to $\left.\theta_{o}\right)$. For $g_{1}=1$ the jamming angle $\theta_{j}=0.3$ (midpoint between $\theta_{o}$ and $\theta_{1}$ ). For very high $g_{1}$ the jamming angle $\theta_{j}=0.5$ (equal to $\theta_{1}$ ). From this figure, it's clear that all curves are mirror imaged at $\phi_{1}=180^{\circ}$ (so that the real of the angle $\theta_{j}$ for $\left(\phi_{1}\right)$ and $\left(360^{\circ}-\phi_{1}\right)$ at constant $g_{1}$ are equal). All curves for $g_{1} \succ 1$, the real of the measured angles are above $0.3^{\circ}$. For $g_{1} \prec 1$, the real of the measured angles are below $0.3^{\circ}$. All these curves for reciprocal values of $g_{1}$ are imaged to each other midpoint between $\theta_{0}$ and $\theta_{1}$.

Now, we study (2) for $n \succ 1$, let $n=2$ in then it can be written as follows 


$$
\theta_{j}=\frac{\theta_{o}+\theta_{1} g_{1} e^{j \phi_{1}}+\theta_{2} g_{2} e^{j \phi_{2}}}{1+g_{1} e^{j \phi_{1}}+g_{2} e^{j \phi_{2}}}
$$

The following discussion is made for the following parameters $\theta_{0}=0.1, \theta_{1}=0.3, \theta_{2}=0.5, g_{1}=0.5$ and $\phi_{1}=30^{\circ}$. We plot the amplitude ratio $g_{2}$ versus the measured angle $\theta_{j}$ for different phase difference $\phi_{2}$. This plot is shown in Fig.9, a zoom in on this figure is shown in Fig.9a. All curves converge to $\theta_{j}=0.5$ for high $g_{2}$ (they tend to reach 0.5 for $g_{2} \succ 10$ ) and for small value of $g_{2}\left(g_{2} \prec 3\right)$; we have high change in the angle $\theta_{j}$.

The relation between the phase differences $\phi_{2}$ versus the measured angle $\theta_{j}$ for different values of $g_{2}$ is shown in Fig.10. This figure differs from Fig.8, for all phase difference from $\left(0^{\circ} \rightarrow 360^{\circ}\right)$ at $g_{2}=0$, the angle $\theta_{j}=$ constant (it is not equal to $\theta_{o}$ ). For very high $g_{2}$ the jamming angle $\theta_{j}=0.5$ (equal to $\theta_{1}$ ). All curves for $g_{1} \succ 1$, the real of the measured angles are above $0.3^{\circ}$. For $g_{1} \prec 1$, the real of the measured angles are below $0.3^{\circ}$.

\section{Performance of the Jamming Under Possible ECCM Techniques}

For a single target, the angle measured by the monopulse tracking radar is real. The presence of jamming signal changes the real part and produces an imaginary part, then the IAC monopulse tracking radar starts to resolve between the real and jamming signal. One of the possible ECCM techniques used against the proposed jammer is called two pulse solution $[3,4]$. To illustrate the idea of this techniques we consider $(n=1)$ in equation (2). Then the complex angle under jamming $\theta_{j}$ is written in the form

$$
\theta_{j}=\frac{\theta_{o}+\theta_{1} g_{1} e^{j \phi_{1}}}{1+g_{1} e^{j \phi_{1}}}
$$

The complex angle $\left(\theta_{j}\right)$ can be written in the form $x+i y$. Separating the complex angle $\theta_{j}$ to a real and imaginary parts as follows,

$$
x+j y=\frac{\theta_{o}+\theta_{1} g_{1}\left[\cos \phi_{1}+j \sin \phi_{1}\right]}{1+g_{1}\left[\cos \phi_{1}+j \sin \phi_{1}\right]}
$$




$$
\begin{array}{r}
x+j y=\frac{\theta_{o}\left(1+g_{1} \cos \phi_{1}+g_{1} \sin \phi_{1}\right)+\theta_{1} g_{1} \cos \phi_{1}\left(1+g_{1} \sin \phi_{1}\right)}{1+g_{1}{ }^{2}+2 g_{1} \cos \phi_{1}} \\
+j \frac{\theta_{1} g_{1} \sin \phi_{1}\left(1+g_{1} \sin \phi_{1}+g_{1} \cos \phi_{1}\right)}{1+g_{1}{ }^{2}+2 g_{1} \cos \phi_{1}}
\end{array}
$$

By equating the real and the imaginary parts of equation (7) in the two hand sides, we get two equations in four unknowns $\left(\theta_{o}, \theta_{1}, g_{1}, \phi_{1}\right)$ and this is only for measuring angle in one direction (azimuth or elevation). If we consider the two directions, we have four equations in six unknowns $\left(\theta_{o a z}, \theta_{o e l}, \theta_{1 a z}, \theta_{1 e l}, g_{1}, \phi_{1}\right)$. These equations can't be solved by only one pulse but requires more than one pulse. Therefore the monopulse radar uses two pulses instead of one to solve this problem, which is not the base of work of monopulse radar. If two-pulses are used, we assume that $\theta_{o}, \theta_{1}$, and $g_{1}$ are the same during the two pulse intervals and there is only a change in $\phi_{1}$ during the first and the second pulses and these values are $\phi_{11}$ and $\phi_{12}$ (subscript $(1,2)$ for $\phi_{1}$ refer to the first and second pulse). The complex measured angle $\left(\theta_{j}\right)$ takes the following two formulas $x_{1}+j y_{1}$ and $x_{2}+j y_{2}$ which represent two points in the complex plane, similar to equation (5) we can represents them as,

$$
\begin{aligned}
& x_{1}+j y_{1}=\frac{\theta_{0}+\theta_{1} g_{1} e^{j \phi_{11}}}{1+g_{1} e^{j \phi_{11}}} \\
& x_{2}+j y_{2}=\frac{\theta_{0}+\theta_{1} g_{1} e^{j \phi_{12}}}{1+g_{1} e^{j \phi_{2}}}
\end{aligned}
$$

These two points determine a unique solution in the complex plane. Note that the center of the circle always lies on the real axis of the complex plane (the three points are enough to determine a unique circle in the complex plane), this circle determines an infinite number of $\theta_{0}, \theta_{1}$ and $g_{1}$. For the solution to be unique, there is an additional consideration as follows, from equations (8) and (9), we have five unknown $\left(\theta_{o}, \theta_{1}, g_{1}, \phi_{11}, \phi_{12}\right)$ in four equation. To have a unique solution we need one more equation, this equation can be obtained from the ratio of the sum channel during the first and the second pulse, which is expressed as

$$
\left|\frac{S_{2}}{S_{1}}\right|^{2}=\frac{1+g_{1}{ }^{2}+2 g_{1} \cos \phi_{12}}{1+g_{1}{ }^{2}+2 g_{1} \cos \phi_{11}}
$$

Although this described method theoretically gives a solution based on only two pulses, some pairs of these pulses will give a good accuracy and others give poor, if 
it happens that $\cos \phi_{11}=\cos \phi_{12}$, the solution is indeterminate. Therefore in practice as many pulses as possible should be used to have a good accuracy for this solution. In equation (2), if we consider $n=2$, the complex angle $\left(\theta_{j}\right)$ is written in the following form

$$
\theta_{j}=\frac{\theta_{0}+\theta_{1} g_{1} e^{j \phi_{1}}+\theta_{2} g_{2} e^{j \phi_{2}}}{1+g_{1} e^{j \phi_{1}}+g_{2} e^{j \phi_{2}}}
$$

then as in equation (8), (9) we have two equations in seven unknowns $\left(\theta_{o}, \theta_{1}, \theta_{2}, g_{1}, g_{2}, \phi_{1}, \phi_{2}\right)$, if we consider the two directions (azimuth and elevation), we have four equations in ten unknowns $\left(\theta_{\text {oat }}, \theta_{\text {oel }}, \theta_{1 a x}, \theta_{1 e l}, \theta_{2 a x}, \theta_{2 e l}, g_{1}, g_{2}, \phi_{1}, \phi_{2}\right)$. The measured complex angle $\left(\theta_{j}\right)$ can be written (as previous) in the form of $\left(x_{1}+j y_{1}\right),\left(x_{2}+j y_{2}\right),\left(x_{3}+j y_{3}\right)$, and $\left(x_{4}+j y_{4}\right)$ where the subscript 1,2,3,4 are referred to the four sequence pulses, These values represent eight equations in thirteen unknowns $\left(\theta_{o}, \theta_{1}, \theta_{2}, g_{1}, g_{2}, \phi_{11}, \phi_{12}, \phi_{13}, \phi_{14}, \phi_{21}, \phi_{22}, \phi_{23}, \phi_{24}\right)$, the additional equations required to provide a unique solution are the ratios of the sum channels, as follows $\left|\frac{S_{2}}{S_{1}}\right|^{2},\left|\frac{S_{3}}{S_{2}}\right|^{2},\left|\frac{S_{3}}{S_{1}}\right|^{2},\left|\frac{S_{4}}{S_{3}}\right|^{2},\left|\frac{S_{4}}{S_{2}}\right|^{2},\left|\frac{S_{4}}{S_{1}}\right|^{2}$

For $n=3$, equation (3) can be written as follows

$$
\theta_{j}=\frac{\theta_{0}+\theta_{1} g_{1} e^{j \phi_{1}}+\theta_{2} g_{2} e^{j \phi_{2}}+\theta_{3} g_{3} e^{j \phi_{3}}}{1+g_{1} e^{j \phi_{1}}+g_{2} e^{j \phi_{2}}+g_{3} e^{j \phi_{3}}}
$$

if we consider six sequenced pulses, we have twenty five unknowns $\left(\theta_{0 \rightarrow 3}, g_{1 \rightarrow 3}, \phi_{11 \rightarrow 16}, \phi_{21 \rightarrow 26}, \phi_{31 \rightarrow 36}\right)$, and the angle $\theta_{j}$ takes six complex values from $\left(x_{1}+j y_{1}\right)$ to $\left(x_{6}+j y_{6}\right)$, which makes twelve equations in additional to equations due to the ratio in sum channel [ $\left|\frac{S_{6}}{S_{5}}\right|^{2}$ to $\left.\left|\frac{S_{6}}{S_{1}}\right|^{2}\right),\left(\left|\frac{S_{5}}{S_{4}}\right|^{2}\right.$ to $\left.\left|\frac{S_{5}}{S_{1}}\right|^{2}\right),\left(\left|\frac{S_{4}}{S_{3}}\right|^{2}\right.$ to $\left.\left|\frac{S_{4}}{S_{1}}\right|^{2}\right),\left(\left|\frac{S_{3}}{S_{2}}\right|^{2}\right.$ to $\left.\left.\left|\frac{S_{3}}{S_{1}}\right|^{2}\right),\left(\left|\frac{S_{2}}{S_{1}}\right|^{2}\right)\right] \quad$ that's make fifteen additional equations, so we have twenty five unknown in twenty seven equations, so it can be solved.

The conclusion of the above discussion is as follows, for $N$ jamming signals, it's required $2 N$ consequence pulses, and these pulses must satisfy that both $\left(\theta_{1}, \theta_{2}, \ldots . ., \theta_{N}\right)$ and their relative amplitude ratios are constant during these pulses duration, which is so difficult to exist in practice because of the motion of the target will change these parameter during these pulses duration. Therefore we will consider $n=2$ in the jamming equation. 


\section{Conclusion}

In the generated deceptive jamming signals, the change in the amplitude ratio makes large effect than the change in the phase difference on the real part of the complex measured error angle. The real value of the complex measured angle due to jamming with constant phase difference and different amplitude ratio has large variation at amplitude ratio less than three. This value converges to the value of the angle of the false target for higher amplitude ratio. There is no jamming effect at amplitude ratios less than one and for some values of phase difference. At these values, the measured angle equals to the angle of the real target. At least two jamming signals are required to have the complex error jamming angle to overcome the ECCM techniques that might be used by the target.

\section{References}

[1] George W. Ewell and Neal T. Alexander, Principle of Modern Radar, Artech house, Inc. 1997

[2] Peyton Z. peebles, JR., Radar Principles, John Wiley and Sons, Inc., 1998.

[3] Sammuel M. Sherman " Complex Indicated Angles Applied to Unresolved Radar Target and Multipath ", IEEE Trans. on Aerospace and Electronic Systems, vol. AES-7, No. 1, January 1971.

[4] Peyton Z. Peebles, JR. and L. Goldman, JR. "Radar Performance with Multipath Using the Complex Angle ", IEEE Trans. on Aerospace and Electronic Systems, vol. AES-7, No. 1. January 1971. 


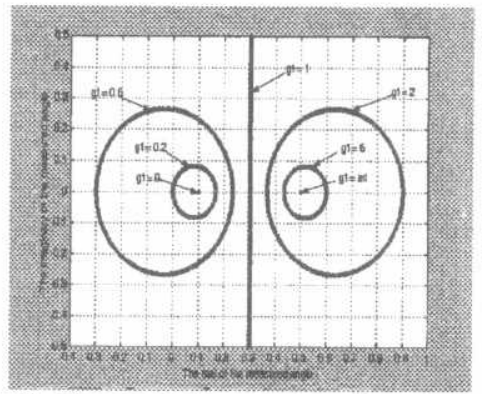

Fig.1. The complex measured angle for different amplitude ratio $g_{1}$

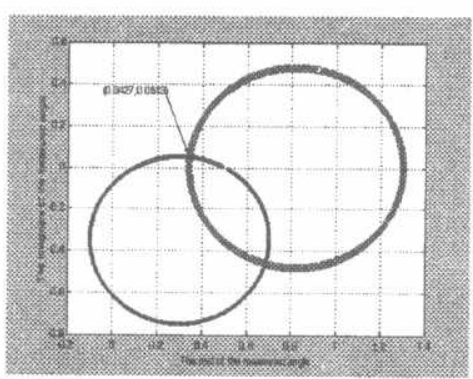

Fig.3. The complex measured angle at

$$
g_{1}=1.5, \phi_{1}=30^{\circ}
$$

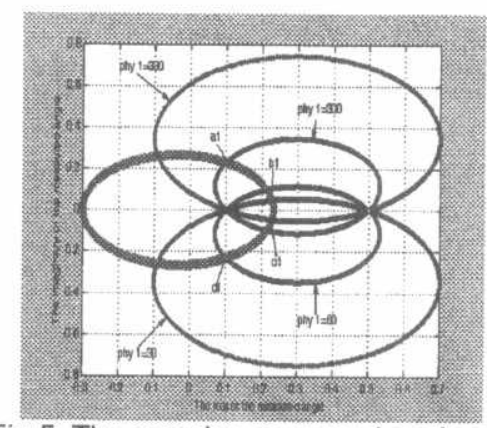

Fig.5. The complex measured angle at different phase difference $\phi_{1}$ and constant amplitude ratio $\left(g_{1}=0.5\right)$

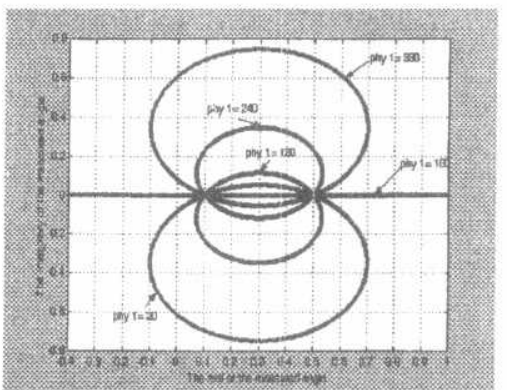

Fig.2. The complex measured angle for constant phase difference $\phi$

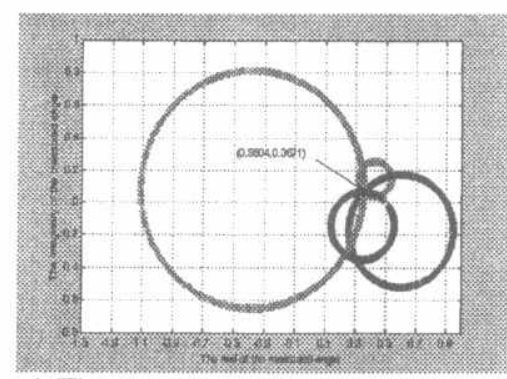

Fig.4. The complex measured angle at $g_{1}=1.5, g_{2}=2, \phi_{1}=30^{\circ}$, and $\phi_{2}=60^{\circ}$

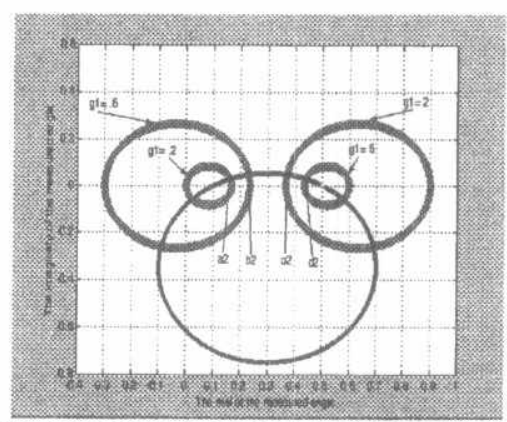

Fig.6. The complex measured angle for different amplitude ratio and constant phase difference $\left(\phi_{1}=30^{\circ}\right)$ 


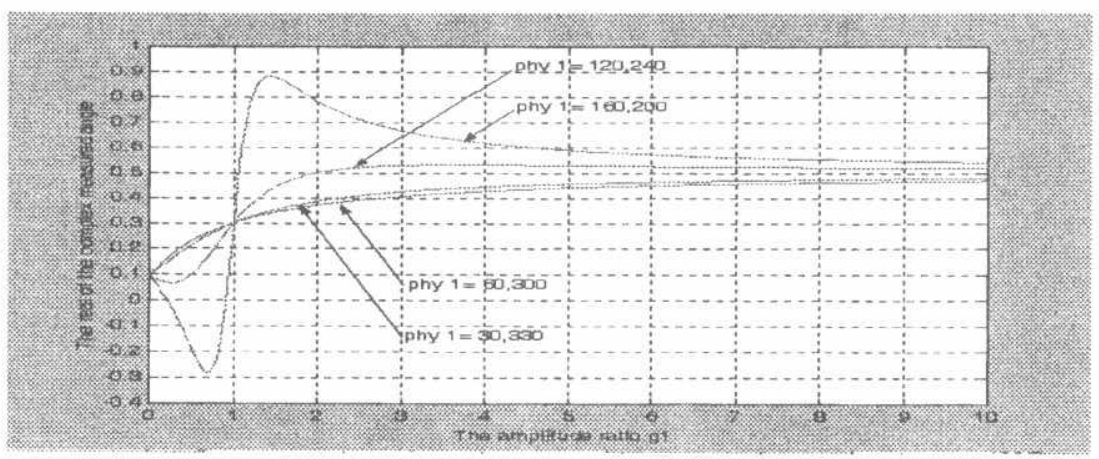

Fig.7. The complex measured angle versus amplitude ratio $g_{1}$

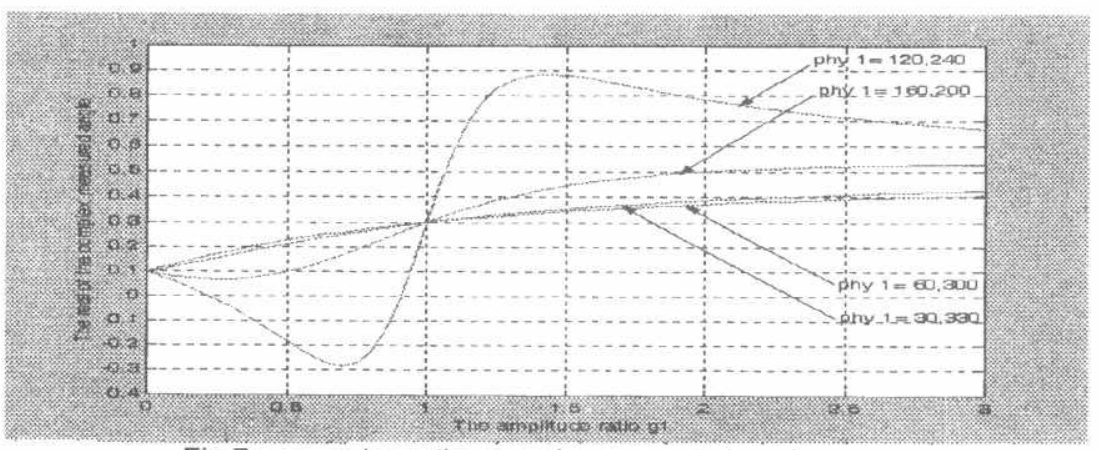

Fig.7a. zoom in on the complex measured angle versus amplitude ratio $g_{1}$

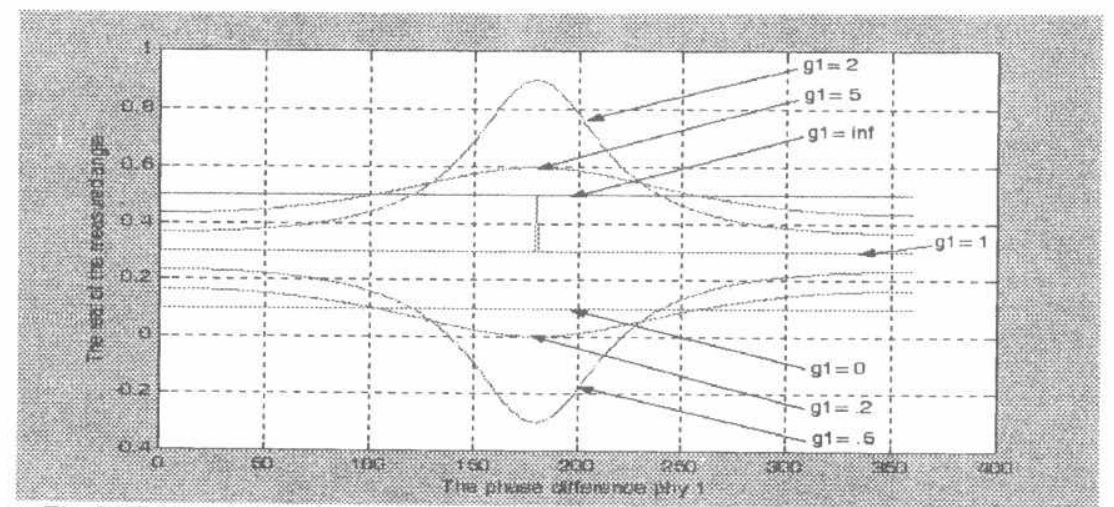

Fig.8. The real of the complex measured angle versus the phase difference for different amplitude ratio $\phi_{1}$. 


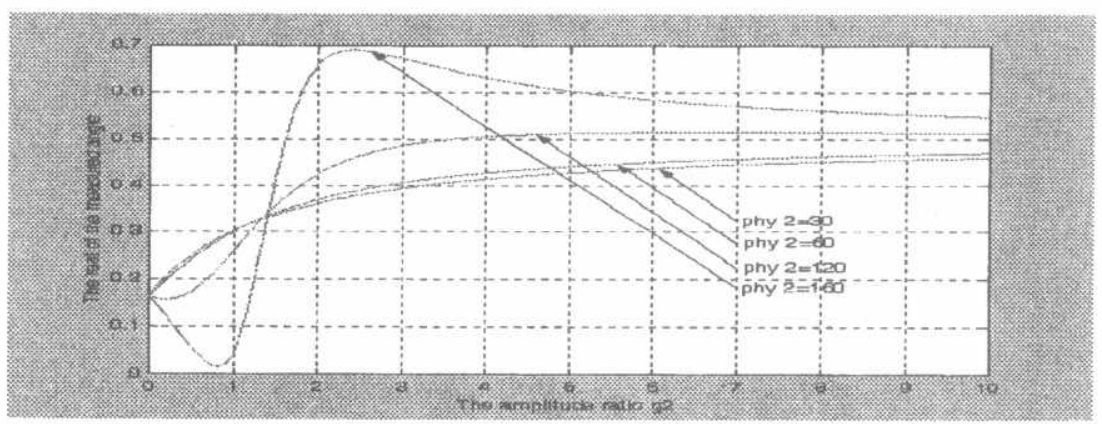

Fig.9. The real of the complex measured angle versus amplitude ratio $g_{2}$ at different constant phase $\phi_{2}$.

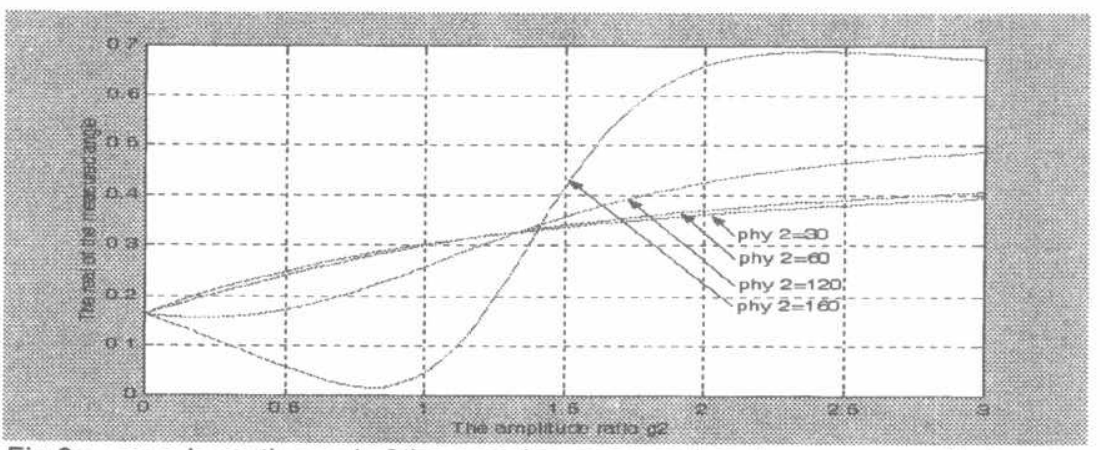

Fig.9a. zoom in on the real of the complex measured angle versus amplitude ratio $g_{2}$ at different constant phase $\phi_{2}$.

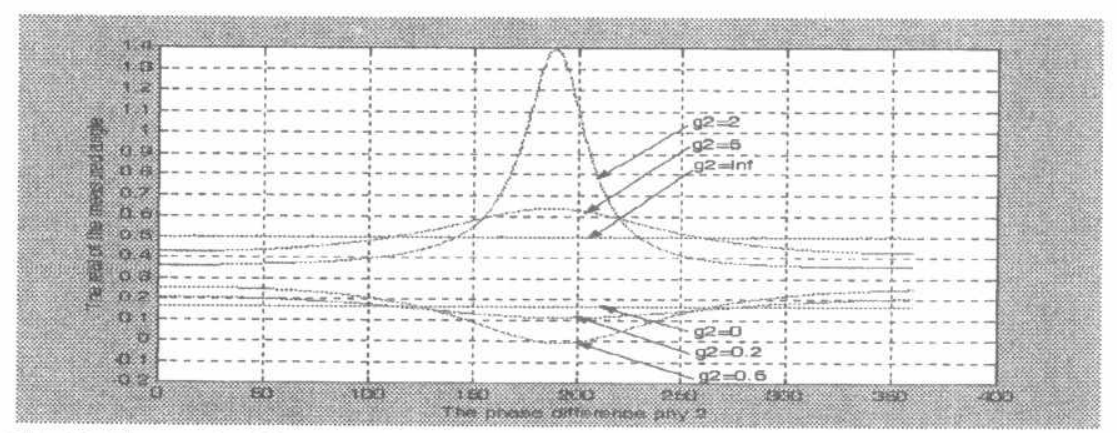

Fig. 10. The real of the complex measured angle versus the phase difference $\phi_{2}$ for different $g_{2}$. 